\title{
Regional cerebral blood flow in late-life depression: arterial spin labelling magnetic resonance study
}

Sean J. Colloby, Michael J. Firbank, Jiabao He, Alan J. Thomas, Akshya Vasudev, Steve W. Parry and John T. O’Brien

\section{Background}

A limited number of studies have demonstrated changes in cerebral blood flow (CBF) in older individuals with depression, but there are considerable inconsistencies between studies.

\section{Aims}

To investigate changes in CBF using arterial spin labelling (ASL) magnetic resonance imaging (MRI) in people with latelife depression and in a similarly aged healthy control group.

\section{Method}

Sixty-eight participants (30 healthy individuals, 38 with depression) underwent ASL and $T_{1}$-weighted MRI scanning. For each individual, regional estimates of separate grey and white matter CBF were obtained. Group differences in CBF and their associations with clinical features were examined.

\section{Results}

Significant increases were observed in white matter CBF in patients with depression relative to the control group $\left(F_{1,65}=9.7, P=0.003\right)$. Grey matter $\mathrm{CBF}$ in lateral frontal, medial frontal, cingulate, central and parietal regions did not significantly differ between groups $\left(F_{1,65} \leqslant 2.1, P \geqslant 0.2\right)$. A significant correlation was found between white matter CBF and Montgomery-Åsberg Depression Rating Scale (MADRS) scores in depression $\left(r^{\prime}=-0.42, P=0.03\right)$. Further analyses revealed that compared with controls, significant elevation of white matter $\mathrm{CBF}$ was apparent in participants whose depression was in remission ( $n=21$, MADRS $\leqslant 10, P=0.001)$ but not in those with current depression $(n=17$, MADRS $\geqslant 11$, $P=0.80$ ).

\section{Conclusions}

Findings suggest a compensatory response to white matter pathological change or a response to (or a predictor of) successful antidepressant treatment, perhaps by facilitating neurotransmission in specific circuits and so reducing depressive symptoms

\section{Declaration of interest}

None.
Depression is common in older people, affecting at least $3 \%$ of people over 65 years old. ${ }^{1}$ It is associated with significant functional impairment and comorbidity, and there is growing evidence that vascular lesions may increase an older person's risk of developing depression., ${ }^{2,3}$ A limited number of functional neuroimaging studies, including single photon emission computed tomography (SPECT) and positron emission tomography (PET), have demonstrated focal or diffuse changes in regional cerebral blood flow $(\mathrm{CBF})$ and cerebral glucose metabolism in older individuals with depression, but there are considerable inconsistencies between studies. In SPECT, regional $\mathrm{CBF}$ deficits in frontal and prefrontal areas appear a consistent finding in people with late-life depression, ${ }^{4-7}$ although anterior cingulate, temporal and parietal regions as well as caudate have also been shown to be implicated. ${ }^{4,5}$ Using PET, greater depressive symptoms were found to be associated with frontal lobe activity in late-life depression. ${ }^{8}$ However, others have found elevated bilateral glucose metabolism in frontal, precuneus and parietal areas in older study participants with depression. ${ }^{9}$

Arterial spin labelling (ASL) magnetic resonance imaging (MRI) is a non-invasive procedure for quantifying CBF. Unlike PET and SPECT techniques, ASL MRI has the advantage of not using radioactive sources or injected contrast agents, allowing for frequent scanning, especially useful in follow-up studies. In addition, anatomical scans can be obtained in the same session, allowing adjustment for effects of atrophy. Evidence from previous functional imaging suggests that frontal, cingulate, parietal and striatal regions may be affected in late-life depression, although there is a major lack of consistency between studies in terms of results and actual brain regions involved. This may be due to differences in samples, depression severity, illness duration or antidepressant medication, as well as in acquisition and analysis methods. Therefore, to help resolve some of the discrepancies in the limited literature in late-life depression and to obtain direct measures of CBF, we applied ASL MRI and region of interest (ROI) methods to investigate grey matter CBF in patients with late-life depression and similarly aged healthy individuals, focusing on specific brain areas including the lateral and medial frontal, cingulate, central and parietal cortices. White matter CBF was also examined, since among the most striking structural features of late-life depression are abnormalities in white matter, with both increased lesions ${ }^{10-12}$ and changes on diffusion imaging. ${ }^{13,14}$ The striatum was not investigated owing to insufficient sampling of this region during the ASL acquisition. The decision to include participants with a current or previous episode of major depression was based on previous structural imaging studies indicating that brain changes are not reversible, are independent of current mood state and pre-date future depressive episodes. ${ }^{2,12,15}$ In view of previous research we predicted that regional CBF changes would be seen in frontal cingulate grey matter and more generalised changes in white matter in late-life depression compared with healthy individuals.

\section{Method}

\section{Participants}

We recruited from secondary care services patients over 60 years old with a history of a DSM-IV major depressive episode, current or previous. ${ }^{16}$ These individuals were assessed by an old age psychiatrist (A.V.) and excluded from the study if they had dementia or a Mini-Mental State Examination (MMSE) score below $24,{ }^{17}$ comorbid or previous drug or alcohol misuse, a history of head injury, epilepsy or cerebrovascular accident, or a myocardial infarction in the preceding 3 months. Healthy older 
individuals with no personal psychiatric history were recruited from the same geographical area. The local research ethics committee approved the study and all participants gave written informed consent. Their assessment included family history of depression, previous psychiatric history, medical history and current medication. Current depression severity was rated using the Montgomery-Åsberg Depression Rating Scale (MADRS) and the 30-item Geriatric Depression Scale (GDS). ${ }^{18,19}$ Other measures recorded included age at onset of depression and duration of depression. Age at onset was determined by interviewing patients and reviewing all available psychiatric records. Duration of depression was ascertained by taking a life history and asking about the time of onset of each depressive episode and time taken for remission for each episode. This was based on information given by the patient and where available, clarification was sought from a partner or caregiver. The duration of each episode was then added up to give a lifetime estimate of duration of depression.

\section{Imaging}

Imaging was performed using a 3.0 T Achieva magnetic resonance scanner (Philips Medical Systems, Eindhoven, The Netherlands). The ASL MRI data were acquired using a multislice pulsed ASL flow-sensitive alternating inversion recovery (FAIR) sequence with six slices. The bottom slice was aligned to mid corpus callosum (Figs 1 and online DS1), no gaps, in-plane resolution $4 \mathrm{~mm}$, slice thickness $6 \mathrm{~mm}$, echo time (TE) $24 \mathrm{~ms}$, repetition time (TR) $4000 \mathrm{~ms}$ and inflow time $1700 \mathrm{~ms}$. In addition to ASL images, $T_{1}$-weighted images covering the whole brain were acquired: magnetisation-prepared rapid gradient echo (MP-RAGE), 150 sagittal slices, $1.2 \times 1.15 \times 1.15 \mathrm{~mm}^{3}$; TR $9.6 \mathrm{~ms}$, TE $4.6 \mathrm{~ms}$, flip angle $8^{\circ}$, SENSE factor 2 . We used $T_{1}$ volumes for image registration, normalisation into standard space, and grey matter and white matter identification.

\section{Data processing and image analysis}

Using SPM8 (www.fil.ion.ucl.ac.uk/spm), the $T_{1}$-weighted threedimensional image was segmented into grey and white matter,

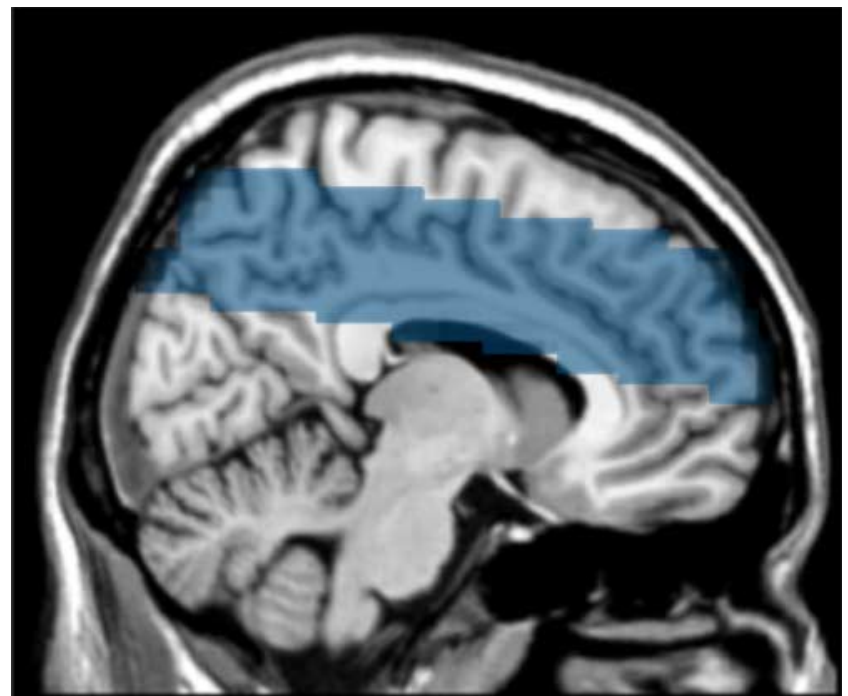

Fig. 1 Sagittal view depicting the acquisition volume.

and coregistered with the mean perfusion image. Perfusion images were transformed to Montreal Neurological Institute space (MNI; www2.bic.mni.mcgill.ca/) using information from their $T_{1}$ coregistration/segmentation. Perfusion volume voxels common to all participants were used to define a mean perfusion mask. From their $T_{1}$ segmentation binary grey matter and white matter masks were created for each participant. Using the SPM toolbox MarsBaR (MARSeille Boîte à Région d'Intérêt, version 0.42, http://marsbar.sourceforge.net/), ROIs were then generated (Figs 2(a) and online DS2(a)). A white matter ROI (Figs 2(b) and online DS2(b)) was also derived from the white matter binary image described by the WFU PickAtlas version 2.4 (www. nitrc.org/projects/wfu_pickatlas/). Last, to reduce type 1 errors, corresponding ROIs from each hemisphere were combined.

From the ASL data we calculated an average and a subtraction image from the labelled and non-labelled scans for each

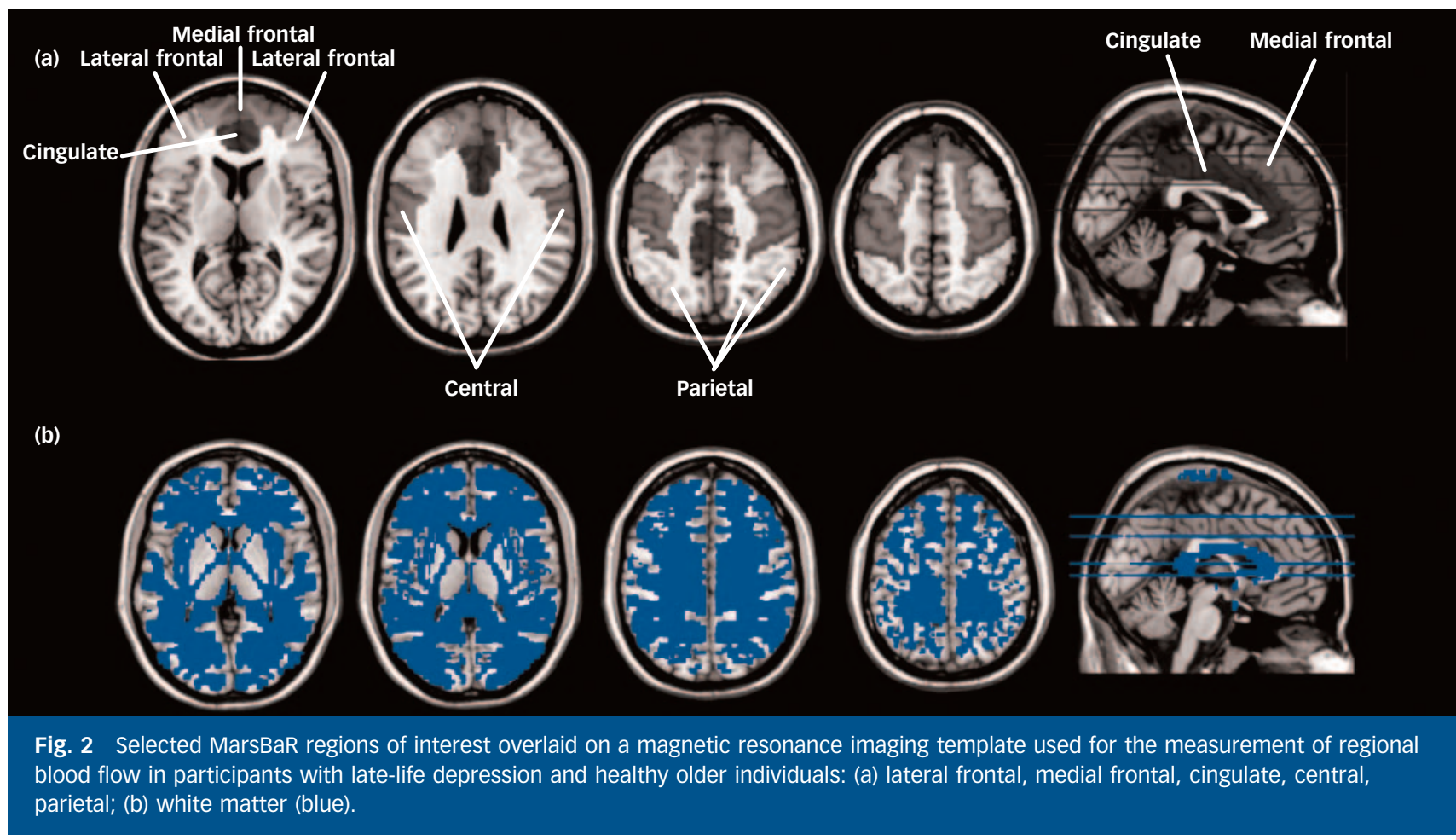


participant; FMRIB's software library (FSL, version 4.01, www.fmrib.ox.ac.uk/fsl/) was used for further image analysis. For grey matter CBF measurements, images were multiplied by the mean perfusion, grey matter and ROI masks, whereas for white matter CBF measurement images were multiplied by the mean perfusion, white matter and ROI white matter masks. Specifically, the combined masks were applied to the average and subtraction ASL images to derive mean values in each ROI. The CBF was then calculated for each ROI from the mean values of average and subtraction ASL images by applying the general kinetic model, ${ }^{20-22}$ using a blood $T_{1}$ of $1490 \mathrm{~ms}$ and blood-brain partition coefficient $(\lambda)$ of 0.9 . To reduce the possible interindividual variability in CBF, we normalised all CBF ROI data by dividing each participant's ROI data by their global mean $\mathrm{CBF}$ value for grey matter.

\section{Statistical analysis}

Where appropriate, differences in demographic and clinical data were assessed using analysis of variance, Mann-Whitney $U$ and chi-squared tests. Group differences in normalised CBF were investigated using analysis of covariance, with imaging parameters as dependent, diagnosis as a fixed factor and age as a covariate. Clinical correlates of normalised CBF were investigated using partial correlation $r^{\prime}$ (age-corrected). A $P$ value of $<0.05$ was considered significant.

\section{Results}

Demographic and clinical characteristics of the sample are given in Table 1. The depression and control groups were comparable for gender, age and total intracranial volume. Thirty-two patients with late-life depression were receiving antidepressants (selective serotonin reuptake inhibitors, venlafaxine, duloxetine or mirtazapine). No patient was taking a tricyclic antidepressant. A third of the currently depressed group $(n=8)$ had had no previous episode of depression; just over half $(54 \%, n=13)$ had experienced one to five lifetime episodes, $4 \%(n=1)$ had six to ten episodes and $8 \%(n=2)$ had experienced more than ten episodes. Using age at onset $<60$ years to define early-onset depression, the early- onset $(n=19)$ and late-onset $(n=19)$ groups were similar in age, in MMSE, GDS and MADRS scores $\left(F_{1,36} \leqslant 1.6, P \geqslant 0.2\right)$ and in gender $\left(\chi^{2}=3.2, P=0.07\right)$.

\begin{tabular}{|c|c|c|c|}
\hline & Control group $(n=30)$ & Depression group $(n=38)$ & Test statistic \\
\hline Gender, $\mathrm{m}: \mathrm{f}: \mathrm{n}$ & $10: 20$ & $11: 27$ & $\chi^{2}=0.2, P=0.7$ \\
\hline Age, years: mean (s.d.) & $74.4(6.4)$ & $74.1(6.1)$ & $F_{1,66}=0.05, P=0.8$ \\
\hline MMSE score, mean (s.d.) & $29.5(0.8)$ & $28.8(1.1)$ & $F_{1,66}=9.02, P=0.004^{* *}$ \\
\hline TIV, $\mathrm{cm}^{3}$ : mean (s.d.) & $1534.9(165.9)$ & $1519.4(162.8)$ & $F_{1,66}=0.2, P=0.7$ \\
\hline MADRS score, mean (s.d.) & NA & $12.9(11.2)$ & \\
\hline GDS score, mean (s.d.) & NA & $11.6(7.9)$ & \\
\hline Age at depression onset, years: mean (s.d.) & NA & $51.8(22.3)$ & \\
\hline Duration of depression, years: mean (s.d.) & NA & $23.0(1.5)$ & \\
\hline \multicolumn{4}{|l|}{ Medication, $n$} \\
\hline Antidepressants & 0 & 32 & \\
\hline SSRIS & 0 & 9 & \\
\hline Venlafaxine & 0 & 14 & \\
\hline Duloxetine & 0 & 3 & \\
\hline Mirtazapine & 0 & 19 & \\
\hline Tricyclics & 0 & 0 & \\
\hline Antihypertensives & 14 & 18 & \\
\hline Diuretics & 6 & 10 & \\
\hline Beta blocker & 7 & 11 & \\
\hline ACE inhibitor & 5 & 10 & \\
\hline ARB & 2 & 2 & \\
\hline Calcium channel blocker & 7 & 5 & \\
\hline Alpha blocker & 0 & 0 & \\
\hline Statins & 10 & 14 & \\
\hline Antiplatelets & 9 & 13 & \\
\hline Warfarin & 3 & 1 & \\
\hline
\end{tabular}

\begin{tabular}{|c|c|c|c|}
\hline & Control group $(n=30)$, mean (s.d.) & Depression group $(n=38)$, mean (s.d.) & Test statistic \\
\hline \multicolumn{4}{|l|}{ Grey matter } \\
\hline Cingulate & $1.163(0.103)$ & $1.134(0.108)$ & $F_{1,65}=1.2, P=0.3^{\mathrm{a}}$ \\
\hline Lateral frontal & $0.944(0.085)$ & $0.941(0.092)$ & $F_{1,65}=0.04, P=0.9^{\mathrm{a}}$ \\
\hline Medial frontal & $0.908(0.129)$ & $0.948(0.099)$ & $F_{1,65}=2.1, P=0.2^{\mathrm{a}}$ \\
\hline Parietal & $0.871(0.097)$ & $0.892(0.132)$ & $F_{1,65}=0.5, P=0.5^{\mathrm{a}}$ \\
\hline Central & $1.018(0.109)$ & $1.008(0.161)$ & $F_{1,65}=0.1, P=0.8^{\mathrm{a}}$ \\
\hline White matter & $0.359(0.093)$ & $0.429(0.095)$ & $F_{1,65}=9.7, P=0.003^{* * a}$ \\
\hline
\end{tabular}




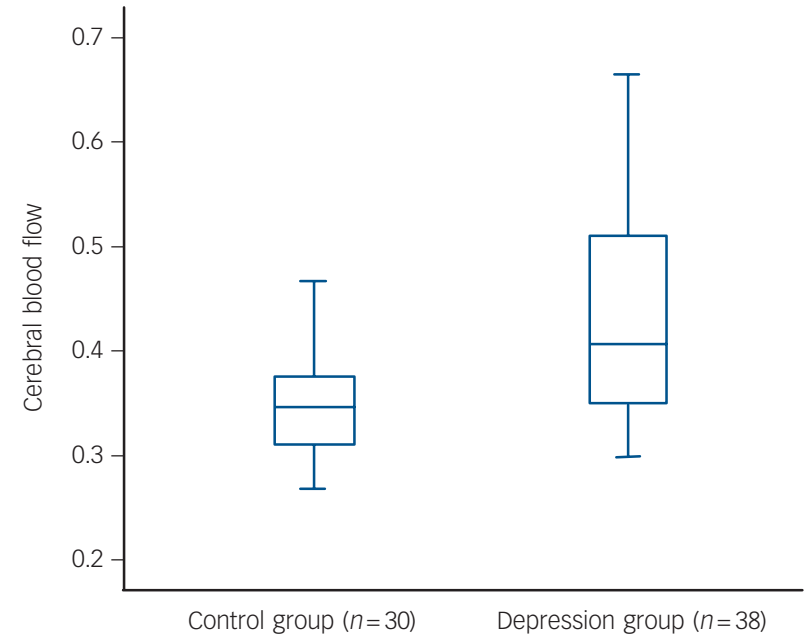

Fig. 3 Box plot of normalised white matter cerebral blood flow in the control group and in participants with late-life depression.

Significant increases in normalised CBF (Table 2) were observed in white matter in patients with late-life depression relative to the control group $\left(F_{1,65}=9.7, P=0.003,19 \%\right.$ increase) . Figure 3 shows the distribution of normalised CBF values in white matter. Grey matter CBF in lateral frontal, medial frontal, cingulate, central and parietal regions did not significantly differ between groups $\left(F_{1,65} \leqslant 2.1, P \geqslant 0.2\right)$. Antidepressant effects on normalised CBF measures were also investigated in the depression group, yielding non-significant results $\left(F_{1,36} \leqslant 1.1, P \geqslant 0.3\right)$. Grey matter and white matter $\mathrm{CBF}$ were also indistinguishable between the early-onset and late-onset groups $\left(F_{1,36} \leqslant 2.4, P \geqslant 0.13\right)$.
Associations between normalised CBF values and key depression variables (MADRS and GDS scores, age at onset of depression and duration of depression) in late-life depression were examined. A significant relationship was found between white matter CBF and MADRS score $\left(r^{\prime}=-0.42, P=0.005\right)$ as well as GDS score $\left(r^{\prime}=-0.32, P=0.03\right)$, but not for age at onset of depression $\left(r^{\prime}=0.06, \quad P=0.4\right)$ or duration of depression $\left(r^{\prime}=-0.06\right.$, $P=0.4)$. Correlations between grey matter CBF and these variables were all non-significant $\left(0.006 \leqslant r^{\prime} \leqslant 0.37, P \geqslant 0.18\right)$. Age and gender were also investigated but did not correlate with white matter or grey matter CBF $(0.003 \leqslant r \geqslant 0.28, P=0.09)$.

Participants with depression were then split into groups: remission $(n=21$, MADRS $\leqslant 10)$ and non-remission $(n=17$, MADRS $\geqslant 11) .{ }^{23}$ These subgroups were comparable in age, gender, MMSE score, age at depression onset and duration of depression (Table 3). Relative proportions taking antidepressant medication were also similar. A significant difference in white matter CBF was observed among depression subgroups (Table 4) and controls $\left(F_{2,64}=8.0, P=0.001\right)$, but not for grey matter CBF $\left(F_{2,64} \leqslant 1.1\right.$, $P \geqslant 0.3)$. Post hoc tests revealed white matter CBF was significantly increased in the remission group compared with controls $(P=0.001)$, suggestive relative to the non-remission group $(P=0.06)$ but similar between non-remission and control groups $(P=0.80)$.

\section{Discussion}

Using the ASL imaging technique we found significant elevation of normalised white matter $\mathrm{CBF}$ in patients with late-life depression compared with healthy older individuals. However, normalised grey matter flow in lateral frontal, medial frontal, cingulate, central and parietal regions did not significantly differ between groups. To our knowledge this is the first report demonstrating increased white matter CBF associated with late-life depression.

Table 3 Characteristics of depression group participants categorised by remission

\begin{tabular}{|c|c|c|c|}
\hline & Remission $^{a}(n=21)$ & Non-remission $(n=17)$ & Test statistic \\
\hline Gender, $\mathrm{m}: \mathrm{f}: \mathrm{n}$ & $6: 15$ & $5: 12$ & $\chi^{2}=0.003, P=0.9$ \\
\hline Age, years: mean (s.d.) & $72.9(5.1)$ & $75.5(7.0)$ & $F_{1,36}=1.8, P=0.2$ \\
\hline MMSE score, mean (s.d.) & $28.7(1.2)$ & $28.9(1.0)$ & $F_{1,36}=0.4, P=0.5$ \\
\hline TIV, $\mathrm{cm}^{3}$ : mean (s.d.) & $1521.6(74.6)$ & $1516.6(152.3)$ & $F_{1,36}=0.009, P=0.9$ \\
\hline MADRS score, mean (s.d.) & $5.2(3.3)$ & $22.3(10.2)$ & $F_{1,36}=52.1, P<0.001 * * *$ \\
\hline GDS score, mean (s.d.) & $6.3(4.9)$ & $18.1(5.9)$ & $F_{1,36}=45.1, P<0.001^{* * *}$ \\
\hline Age at depression onset, years: mean (s.d.) & $46.1(22.9)$ & $57.2(20.6)$ & $F_{1,36}=2.4, P=0.1$ \\
\hline Duration of depression, years: mean (s.d.) & $26.8(22.0)$ & $18.3(20.6)$ & $F_{1,36}=1.5, P=0.2$ \\
\hline \multicolumn{4}{|l|}{ Medication, $n$} \\
\hline Antidepressants & 16 & 16 & \\
\hline SSRIS & 3 & 6 & \\
\hline Venlafaxine & 6 & 8 & \\
\hline Duloxetine & 3 & 0 & \\
\hline Mirtazapine & 8 & 11 & \\
\hline Tricyclics & 0 & 0 & \\
\hline Antihypertensives & 9 & 9 & \\
\hline Diuretics & 5 & 5 & \\
\hline Beta blocker & 8 & 3 & \\
\hline ACE inhibitor & 5 & 5 & \\
\hline ARBS & 0 & 2 & \\
\hline Calcium channel blocker & 1 & 4 & \\
\hline Alpha blocker & 0 & 0 & \\
\hline Statins & 7 & 7 & \\
\hline Antiplatelets & 6 & 7 & \\
\hline Warfarin & 1 & 0 & \\
\hline \multicolumn{4}{|c|}{$\begin{array}{l}\text { ACE, angiotensin converting enzyme; ARB, angiotensin II receptor blocker; GDS, Geriatric Depression Scale; MADRS, Montgomery-Åsberg Depression Rating Scale; MMSE, Mini- } \\
\text { Mental State Examination; TIV, total intracranial volume; SSRI, selective serotonin reuptake inhibitor. } \\
\text { a. Remission defined as an MADRS score of } 10 \text { or below. }{ }^{*} P<0.05,{ }^{*} P<0.01,{ }^{* * * P<0.001 .}\end{array}$} \\
\hline
\end{tabular}


Table 4 Regional cerebral blood flow volumes, normalised to mean global grey matter flow, in depression group participants categorised by remission state

\begin{tabular}{|c|c|c|}
\hline & $\begin{array}{l}\text { Remission group } \\
\qquad(n=21) \\
\text { Mean (s.d.) }\end{array}$ & $\begin{array}{l}\text { Non-remission group } \\
\qquad(n=17) \\
\text { Mean (s.d.) }\end{array}$ \\
\hline \multicolumn{3}{|l|}{ Grey matter } \\
\hline Cingulate & $1.135(0.126)$ & $1.134(0.084)$ \\
\hline Lateral frontal & $0.926(0.099)$ & $0.960(0.080)$ \\
\hline Medial frontal & $0.939(0.082)$ & $0.959(0.119)$ \\
\hline Parietal & $0.901(0.147)$ & $0.881(0.114)$ \\
\hline Central & $1.032(0.180)$ & $0.978(0.135)$ \\
\hline White matter & $0.456(0.093)$ & $0.395(0.090)$ \\
\hline
\end{tabular}

Younger adults with depression have shown increased CBF in frontal, central, temporal and parietal regions compared with healthy adults when ROI methods are used, although such procedures cannot determine separate flow in grey matter or white matter. $^{24}$ Others have reported elevated cerebral glucose metabolism in frontal, precuneal and parietal areas in older patients with depression, ${ }^{9}$ although this was associated with atrophy in the same regions. As well as increased white matter lesions, impairments to white matter connectivity have also been described in late-life depression using diffusion tensor imaging. ${ }^{13,14}$ Further investigations are required in order to understand the importance of such white matter changes and their influence on depressive symptoms in late-life depression. Our study included participants with depression and those who had recovered, with the mean MADRS score of 12.9 reflecting this.

In the late-life depression group a highly significant correlation was found between white matter CBF and MADRS score. Therefore CBF results may have been influenced by the current mood state of people in this group. In an attempt to investigate this issue further, the cohort was dichotomised into those with remitted and non-remitted depression. Elevation of white matter $\mathrm{CBF}$ was apparent in the remission group relative to the non-remission and control groups. Clinical characteristics were broadly similar in the two depression subgroups. Results may suggest that increased white matter CBF could be either a response to, or a predictor of, successful antidepressant treatment, perhaps by facilitating neurotransmission in specific circuits and so reducing depressive symptoms.

Late-life depression research has mainly focused on studying structures implicated within the limbic-cortical-striatal-pallidalthalamic circuit. White matter hyperintensities and abnormalities of white matter tracts may indicate disturbances to specific neural circuits or fibre tracts involved in mood regulation. Increased white matter $\mathrm{CBF}$ could represent a response to white matter pathological change or be a marker of those who respond to treatment. However, the latter theory would need to be tested in prospective studies.

We acknowledge that results specifically in grey matter $\mathrm{CBF}$ may have been underpowered owing to the heterogeneity of our depression cohort, almost two-thirds of whom were in a currently depressed state. Other limitations of our study include depressive symptoms in controls and striatal CBF not being measured. In conclusion, our findings combined with previous evidence showing increased white matter lesions and both connectivity and diffusion changes, further suggest that white matter changes may be more relevant than those in grey matter for explaining the underlying neurobiology of late-life depression. Future studies investigating functional imaging changes in the depressed and recovered state of late-life depression are required.
Sean J. Colloby, PhD, Michael J. Firbank, PhD, Jiabao He, PhD, Alan J. Thomas $\mathrm{PhD}$, Akshya Vasudev, MD, Steve W. Parry, PhD, John T. O'Brien, DM, Institute for Ageing and Health, Newcastle University, Newcastle upon Tyne, UK

Correspondence: Dr Sean J. Colloby, Institute for Ageing and Health, Campus for Ageing and Vitality, Newcastle University, Newcastle upon Tyne NE4 5PL, UK. Email: s.j.colloby@ncl.ac.uk

First received 28 Jan 2011, final revision 22 Jul 2011, accepted 15 Sep 2011

\section{Funding}

This work was supported by the UK National Institute for Health Research Biomedical Research Centre for Ageing and Age-related Disease award to the Newcastle upon Tyne Hospitals National Health Service Foundation Trust.

\section{Acknowledgements}

We thank all participants for their invaluable contribution.

\section{References}

1 Beekman AT, Copeland JR, Prince MJ. Review of community prevalence of depression in later life. Br J Psychiatry 1999; 174: 307-11.

2 Teodorczuk A, Firbank MJ, Pantoni L, Poggesi A, Erkinjuntti T, Wallin A, et al. Relationship between baseline white-matter changes and development of late-life depressive symptoms: 3-year results from the LADIS study. Psychol Med 2010; 40: 603-10.

3 Vaishnavi S, Taylor WD. Neuroimaging in late-life depression. Int Rev Psychiatry 2006; 18: 443-51.

4 Awata $\mathrm{S}$, Ito $\mathrm{H}$, Konno M, Ono S, Kawashima R, Fukuda H, et al. Regional cerebral blood flow abnormalities in late-life depression: relation to refractoriness and chronification. Psychiatry Clin Neurosci 1998; 52 97-105.

5 Ishizaki J, Yamamoto $\mathrm{H}$, Takahashi T, Takeda M, Yano M, Mimura M. Changes in regional cerebral blood flow following antidepressant treatment in late-life depression. Int J Geriatr Psychiatry 2008; 23: 805-11.

6 Navarro V, Gasto C, Lomena F, Mateos JJ, Marcos T. Frontal cerebral perfusion dysfunction in elderly late-onset major depression assessed by 99MTC-HMPAO SPECT. Neuroimage 2001; 14: 202-5.

7 Nobler MS, Roose SP, Prohovnik I, Moeller JR, Louie J, Van Heertum RL, et al. Regional cerebral blood flow in mood disorders, V.: Effects of antidepressant medication in late-life depression. Am J Geriatr Psychiatry 2000; 8: 289-96

8 Dotson VM, Beason-Held L, Kraut MA, Resnick SM. Longitudinal study of chronic depressive symptoms and regional cerebral blood flow in older men and women. Int J Geriatr Psychiatry 2009; 24: 809-19.

9 Smith GS, Kramer E, Ma Y, Kingsley P, Dhawan V, Chaly T, et al. The functional neuroanatomy of geriatric depression. Int J Geriatr Psychiatry 2009; 24: 798-808.

10 Herrmann LL, Le Masurier M, Ebmeier KP. White matter hyperintensities in late life depression: a systematic review. J Neurol Neurosurg Psychiatry 2008; 79: 619-24.

11 O'Brien J, Desmond P, Ames D, Schweitzer I, Harrigan S, Tress B. A magnetic resonance imaging study of white matter lesions in depression and Alzheimer's disease. Br J Psychiatry 1996; 168: 477-85.

12 Teodorczuk A, O’Brien JT, Firbank MJ, Pantoni L, Peggesi A, Erkinjuntti T, et al. White matter changes and late-life depressive symptoms. Longitudinal study. Br J Psychiatry 2007; 191: 212-7.

13 Shimony JS, Sheline YI, D'Angelo G, Epstein AA, Benzinger TL, Mintun MA, et al. Diffuse microstructural abnormalities of normal-appearing white matter in late life depression: a diffusion tensor imaging study. Biol Psychiatry 2009; 66: $245-52$.

14 Yang Q, Huang X, Hong N, Yu X. White matter microstructural abnormalities in late-life depression. Int Psychogeriatr 2007; 19: 757-66.

15 Godin O, Dufouil C, Maillard P, Delcroix N, Mazoyer B, Crivello F, et al. White matter lesions as a predictor of depression in the elderly: the 3C-Dijon study. Biol Psychiatry 2008; 63: 663-9.

16 American Psychiatric Association. Diagnostic and Statistical Manual of Mental Disorders (4th edn) (DSM-IV). APA, 1994. 
17 Folstein MF, Folstein SE, McHugh PR. 'Mini-mental state'. A practical method for grading the cognitive state of patients for the clinician. J Psychiatr Res 1975: 12: 189-98.

18 Montgomery SA, Åsberg M. A new depression scale designed to be sensitive to change. Br J Psychiatry 1979; 134: 382-9.

19 Yesavage JA. Geriatric Depression Scale. Psychopharmacol Bull 1988; 24 709-11.

20 Buxton RB, Frank LR, Wong EC, et al. A general kinetic model for quantitative perfusion imaging with arterial spin labeling. Magn Reson Med 1998; 40: 383-96.

21 Kim SG. Quantification of relative cerebral blood flow change by flowsensitive alternating inversion recovery (FAIR) technique: application to functional mapping. Magn Reson Med 1995; 34: 293-301.
22 Kim SG, Tsekos NV. Perfusion imaging by a flow-sensitive alternating inversion recovery (FAIR) technique: application to functional brain imaging Magn Reson Med 1997; 37: 425-35.

23 Zimmerman M, Posternak MA, Chelminski I. Derivation of a definition of remission on the Montgomery-Åsberg depression rating scale corresponding to the definition of remission on the Hamilton rating scale for depression. J Psychiatr Res 2004; 38: 577-82.

24 Pagani M, Gardner A, Salmaso D, Sánchez Crespo A, Jonsson C, Jacobsson H, et al. Principal component and volume of interest analyses in depressed patients imaged by 99mTC-HMPAO SPET: a methodological comparison. Eur J NuCl Med Mol Imaging 2004; 31: 995-1004.

\section{Swaran P. Singh}

The history of humanity is a story of migrants. Gradual migration was the progenitor of trade, innovations, war and epidemics. Recent rapid migration across vast cultural landscapes can bewilder both migrants and hosts. Migrants experience contrary emotions juxtapositioned: grief and renewal, loss and hope, separation and assimilation. The host feels invaded. The results, positive health or mental illness, arise from this complex, multilayered interplay - there is no inevitability of outcome. Our relationship with land is intergenerational: the land where our ancestors lived and which our children inherit. As migrants develop such cross-generational links with their new land, flourishing replaces distress. 